Yelle (NASA/Ames, Moffett Field), C. Barnet (Space Astrophys. Lab., North York, Ontario), S. Edgington (U. Michigan, Ann Arbor)

The composition of Jupiter's stratosphere and upper troposphere was dramatically altered by the impacts of the fragments of comet P/ShoemakerLevy 9. UV spectra obtained with the Hubble Space Telescope have identified at least ten molecules and atoms in the vicinity of impact sites, most never before observed in Jupiter. The large mass of sulfur-containing material, more than $10^{14} \mathrm{~g}$ in $\mathrm{S}_{2}$ alone, and the relative abundances of sulfur-bearing gases, indicates that many of the sulfur-containing molecules $\mathrm{S}_{2}, \mathrm{CS}_{2}, \mathrm{CS}, \mathrm{H}_{2} \mathrm{~S}$, and $\mathrm{S}^{+}$may be derived from a sulfur-bearing parent molecule native to Jupiter. If so, this is the first observation of any sulfur compound in Jupiter and is an observational confirmation of models of cloud composition and possibly cloud coloration. The presence of jovian sulfur also constrains the depth of penetration of the fragments with the requirement that at least some must reach the expected $\mathrm{NH}_{4} \mathrm{SH}$ cloud at $\mathrm{P} \sim 1-2$ bars. For the sulfur to be derived from the impactor, a mass equivalent to a $3 \mathrm{~km}$ diameter comet or asteroid must be deposited in Jupiter's stratosphere above 100 mbar. The presence of stratospheric $\mathrm{NH}_{3}$ is also consistent with relatively deep penetration of the impactors. Neutral and ionized metals were observed in emission, including MgII, MgI, SiI, FeI, and FeII. The mass required is easily derived from a modest sized impactor. Oxygen-containing molecules were significant by their absence with the possible significant exception of $\mathrm{H}_{2} \mathrm{O}$. We derive upper limits for $\mathrm{SO}_{2}, \mathrm{SO}, \mathrm{CO}, \mathrm{SiO}$, and $\mathrm{H}_{2} \mathrm{O}$.

\title{
W. M. KECK TELESCOPE OBSERVATIONS OF THE COMET P/SHOEMAKER-LEVY 9 FRAGMENT R JUPITER COLLISION
}

I. de Pater, J.R. Graham, J.G. Jernigan, M.C. Liu, M.E. Brown, (U. California, Berkeley, USA)

We used the W. M. Keck telescope in Hawaii, the largest optical telescope in the world ( $10 \mathrm{~m}$ diameter) to observe the impacts of Comet P/ShoemakerLevy 9 with Jupiter. Our most surprising result was the impact of fragment $R$, which we recorded in the infrared at $2.3 \mu \mathrm{m}$ on July $21 \mathrm{UT}$. Our data, which consist of a sequence of images every $7.7 \mathrm{~s}$, show three outbursts. The first flash lasts about $40 \mathrm{~s}$, and is followed one minute after its peak by a second flash which lasts about three minutes. A third, longer lasting flare, begins brightening six minutes after the first flash, and lasts for ten minutes. At its maximum brightness the flare outshines Jupiter at this wavelength. All events 
occur on the limb of Jupiter at the latitude of the expected impact site. The two short flashes are probably associated with the initial meteor streak and the subsequent fireball, respectively. The bright flare occurs when the impact site rotates into view. If this interpretation is correct then fragment $\mathrm{R}$ collided with Jupiter at 05:34:45 UT.

Our observations suggest the plume to reach altitudes maybe many hundreds of kilometers above the visible cloud tops. The luminosity of the impact site during the long bright flare is probably maintained by the release of gravitational potential energy as plume material falls back onto the lower atmosphere. Our results agree qualitatively with the predictions for the explosion of $\mathrm{a} \approx 0.5 \mathrm{~km}$ diameter comet fragment which releases $\approx 10^{27} \mathrm{ergs}$ in the Jovian atmosphere at a depth corresponding to a pressure of about 2 bars.

\section{NEAR-IR SPECTROSCOPIC MONITORING OF JUPITER AT THE TIME OF THE SL-9 IMPACT}

R. Schulz (MPAE, Germany), Th. Encrenaz (Obs. Paris, France), J.A. Stüwe (MPAE, Germany), G. Wiedemann (ESO)

Jupiter was monitored spectroscopically in the near-IR with the IRSPEC imaging spectrograph mounted on the $3.5 \mathrm{~m}$ New Technology Telescope at ESO, La Silla, Chile, between 16 and 28 July 1994 (except for 19 to 21 July due to bad weather) with a final observing night on July 30-31. During the week of impacts the 4".4 slit was aligned along the parallel of the impact sites (lat $=-44^{\circ}$ ). This allowed to search into immediate implications of each impact to the Jovian atmosphere at the time of event and to study the evolution of the older impact sites additionally present in the spectra. Starting on $23 \mathrm{July}$, we also observed the counterpart of the impact region in the northern hemisphere (lat $=+44^{\circ}$ ) and proceeded with a mapping of the entire planet. Data were recorded in several spectral regions between $2 \mu \mathrm{m}$ and $5 \mu \mathrm{m}$ with preference to the regions around the $\mathrm{H}_{3}^{+}$multiplet at $3.5 \mu \mathrm{m}$ and the $\mathrm{H}_{2} \mathrm{~S}(1)$ line at $2.12 \mu \mathrm{m}$. Fragments $\mathrm{B}, \mathrm{F}$ and $\mathrm{H}$ were observed at time of impact.

Before and during the impact of fragment $B$ on July 16 (0:21-3:58 UT) spectra were recorded in the $2.12 \mu \mathrm{m}$ range. The spectra do not show any effects of impact B to the Jovian atmosphere. However, they also cover impact site $A$ (approx. 5h after impact), where the emission of the $\mathrm{H}_{2} \mathrm{~S}(1)$ quadrupole line was detected. From the $\mathrm{H}_{2}$ observations recorded shortly after impact $\mathrm{F}$ on $18 \mathrm{July}, 2: 30 \mathrm{UT}$, we are able to distinguish between the impact sites $\mathrm{E}$ and $F$ that lie side by side very close together. The spectrum of $F$ is characterized 